\title{
Work in Progress: A Multidisciplinary Approach for Undergraduate Re- search in Augmented Reality Systems
}

\section{Prof. Yuzhong Shen, Old Dominion University}

Yuzhong Shen received his B.S. degree in Electrical Engineering from Fudan University, Shanghai, China, M.S. degree in Computer Engineering from Mississippi State University, Starkville, Mississippi, and $\mathrm{Ph} . D$. degree in Electrical Engineering from the University of Delaware, Newark, Delaware. His research interests include computer graphics, visualization, serious games, signal and image processing, and modeling and simulation. Dr. Shen is currently Professor of the Department of Modeling, Simulation, and Visualization Engineering and the Department of Electrical and Computer Engineering of Old Dominion University. He is also affiliated with Virginia Modeling, Analysis, and Simulation Center (VMASC). Dr. Shen is a Senior Member of IEEE.

\section{Dr. Anthony W. Dean, Old Dominion University}

Dr. Anthony W. Dean has had several roles in academia. He is currently Assistant Dean for Research, Batten College of Engineering and Technology (BCET) at ODU. His previous appointments include Associate Professor of Engineering Technology and as Associate Director of the Institute for Ship Repair, Maintenance, and Operations at Old Dominion University (ODU).His research has focused mostly on control systems (integration and testing) and the reliability and maintainability of complex systems. He has been selected as both a NASA and an ONR Faculty Fellow. He regularly teaches courses in Marine Engineering and in Maintained Systems. Most recently Dr. Dean was on the Headquarters Staff the American Society of Naval Engineers. He received his Ph.D. from the Department of Engineering Management and Systems Engineering, and a B.S. in Nuclear Engineering Technology, from the Batten College of Engineering and Technology at Old Dominion University. Additionally, Dr. Dean received an MBA from the College of William and Mary. Prior to is academic career Dr. Dean was Director of Operations and Business Development for Clark-Smith Associates, P.C., and served as an Electrician in the US Navy aboard the USS South Carolina and the USS Enterprise.

\section{Xiaoyu Zhang}

Dr. Xiaoyu Zhang's research interests include high-temperature electrolysis, fuel cell, photoelectrochemical water splitting, 3D printing.

\section{Dr. Rafael E. Landaeta, Old Dominion University}

Dr. Landaeta is an Associate Professor of Engineering Management and Systems Engineering at Old Dominion University in Norfolk, Virginia. He holds a Ph.D. in Industrial Engineering and an M.S. in Engineering Management from the University of Central Florida, as well as, a B.S. in Mechanical Engineering from UNITEC Venezuela. Dr. Landaeta serves as the Associate Dean for Undergraduate Programs at the Batten College of Engineering \& Technology.

Edwin Merino, Old Dominion University Jayson Carl Alberto Kreger 


\title{
Work-in-Progress: A Multidisciplinary Approach for Undergraduate Research in Augmented Reality Systems
}

\begin{abstract}
This Work-in-Progress paper presents a multidisciplinary undergraduate research project to develop an augmented reality system for the U.S. Marine Corps weapon maintenance and operation. The project utilizes low-cost, market-leading AR hardware and software to develop an interactive AR application for maintenance and operation of M16A4 rifle. The AR application contains interactive presentation and visualization of M16A4 external and internal parts and 3D animations for maintenance and troubleshooting procedures. The proposed approach can be applied to other similar weapons/equipment with minimal changes. The project will also conduct preliminary research on software framework, interface, and file structures for easy integration of different objects under the same AR application.
\end{abstract}

This project contains several major tasks: 1) 3D modeling of the weapon, including all internal and external parts to be displayed in the AR application, 2) 3D printing of the weapon mockup that only requires the external parts converted from the file format used in Task 1 and further optimization of the model for 3D printing, 3) software development in Unity that utilizes mobile devices, Microsoft HoloLens, and Vuforia to generate the AR application for weapon maintenance and operation, and 4) preliminary research on software and information architecture to support efficient development of AR applications. This paper discusses the issues, benefits, and effective approaches in designing and executing multidisciplinary undergraduate research projects.

\section{Introduction}

Augmented Reality (AR) augments the real world by overlaying information and/or virtual objects that are spatially aligned to the objects in the real world [1,2]. With recent advances in computing hardware and software technology, AR is making gradual but solid progress in a wide range of fields, such as manufacturing, construction, maintenance, training, and healthcare. Started with head mounted displays, now AR technologies are available on a variety of platforms, such as mobile devices and heads-up displays (HUD). The enormously successful game Pokemon Go is an AR game that uses mobile devices to locate, capture, battle, and train virtual creatures, Pokemon, which appear as if they are in the player's real-world location [3]. Starting from May 2018, all new passenger vehicles sold in U.S. must have backup cameras, which contain onscreen (augmented) guidelines to assist the driver with gauging distance from objects in the vehicle's path. The significant advances in AR hardware are echoed by the major progress in software libraries and tools for AR application development in recent years. Major mobile platforms now provide application programming interfaces (APIs) for handheld AR App development, such as ARKit for iOS [4] and ARCore for Android [5]. Advanced 3D game and simulation development tools such as Unity [6] and Unreal [7] and advanced target recognition and tracking software libraries such as Vuforia [8,9] and ARToolkit considerably lower the barriers to AR development, leading to rapid development of high-quality AR applications.

Modern engineering systems and applications comprise of various types of subsystems or components that entail expertise from different disciplines. For instance, automobile design involves industrial design, mechanical engineering, electrical engineering, computer engineering, 
computer science, human factors, and psychology, among other fields. Various studies and reports underscore the importance of incorporating multidisciplinary approaches in curriculum and workforce development [10-17]. The ability to function on multidisciplinary teams is one of the core student outcomes assessed by The Accreditation Board for Engineering and Technology (ABET) for engineering degree programs [18]. This paper describes a multidisciplinary undergraduate research project to develop an augmented reality system for the U.S. Marine Corps weapon maintenance and operation. The project utilizes low-cost, marketleading AR hardware and software, e.g., mobile devices, Microsoft HoloLens, and Vuforia, to develop an interactive AR application for maintenance and operation of M16A4 rifle used by the U.S. Marine Corps. The AR application contains interactive presentation and visualization of M16A4 external and internal parts and 3D animations for maintenance and troubleshooting procedures. The multidisciplinary project team includes two undergraduate students and two faculty members from three academic departments: Department of Modeling, Simulation, and Visualization Engineering (MSVE), Department of Mechanical and Aerospace Engineering (MAE), and Department of Electrical and Computer Engineering. This project is most relevant to the following new ABET student outcomes: Outcome 3 - an ability to communicate effectively with a range of audiences, Outcome 5 - an ability to function effectively on a team whose members together provide leadership, create a collaborative and inclusive environment, establish goals, plan tasks, and meet objectives, and Outcome 7 - an ability to acquire and apply new knowledge as needed, using appropriate learning strategies. This paper discusses the issues, benefits, and effective approaches in designing and executing multidisciplinary undergraduate research projects.

The remainder of this paper is organized as follows: Section 2 introduces Marine Corps weapon operation and maintenance. Section 3 describes the design and implementation of the AR project for M16A4. Section 4 presents current results. Section 5 discusses future work and challenges.

\section{Marine Corps Weapon Operation and Maintenance}

One important use of augmented reality is equipment operation and maintenance $[1,2]$. U.S. Marine Corps operate a wide range of weapons and equipment with ever increasing sophistication. To ensure combat readiness, weapons and equipment must be properly maintained in operating condition to perform designated functions effectively. U.S. Marine Corps Warfighting Publication (MCWP) 4-11.4, Maintenance Operations [19], provides the commander with the basic information on the Marine Corp's maintenance system and how the system is employed to maintain a unit's equipment in a combat-ready condition.

The basic infantry weapon of U.S. Marine Corps is automatic rifles. The M16A4 is the $4^{\text {th }}$ generation of the M16 series and it was adopted by the Marine Corps in 1998. As Marine's standard weapon, proper maintenance of M16A4 rifles is critical to Marine's combat performance and safety. The U.S. Marine Corps has published a technical manual for maintenance of M16A4, Organizational and Intermediate Maintenance Manual with Repair Parts List (RPL) for M16A2, M16A4, M4, and M4A1 [20], which comprises 370 pages and contains information on general equipment description and data, principles of operation, troubleshooting, organizational maintenance, etc. Figure 1 shows two excerpts from the manual: Figure 1(a) shows an inspection procedure during cleaning and Figure 1(b) shows the steps in 
reassembly. The maintenance manual for M16A4 [3] is in PDF format and mainly contains text and figures.

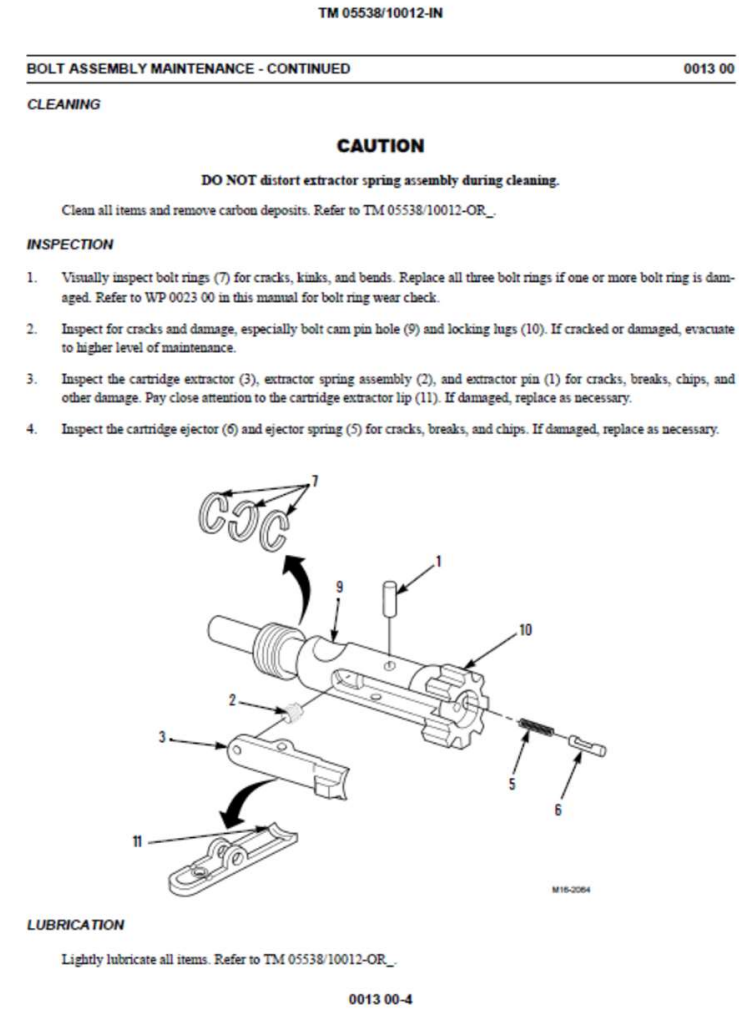

(a)

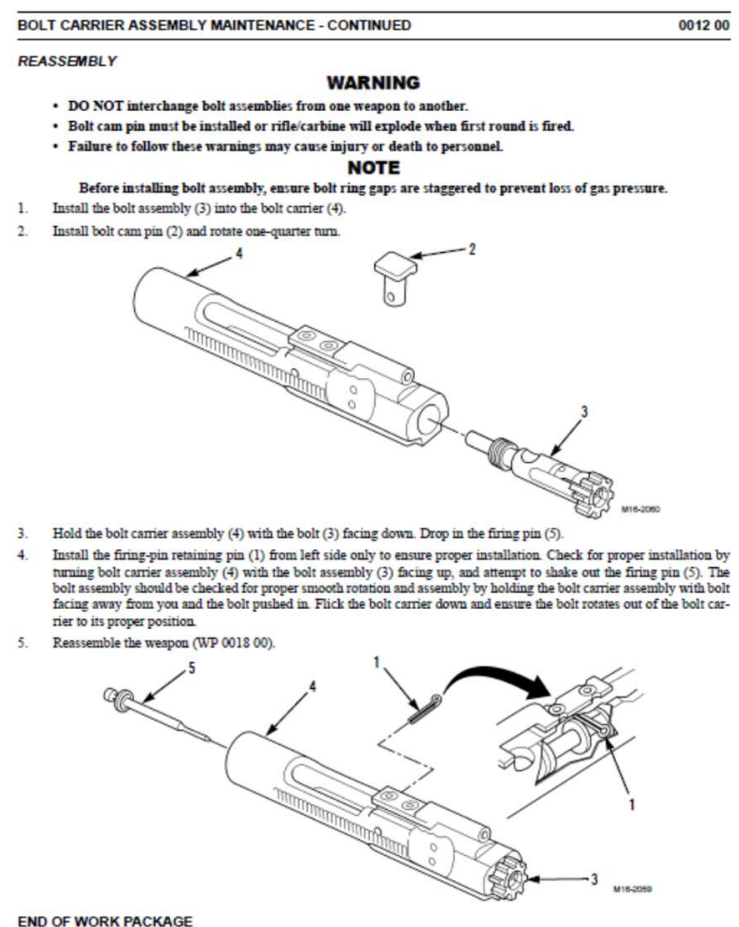

$001200-4$

(b)

Figure 1. Excerpts from Organizational and Intermediate Maintenance Manual of M16A4 [20]. (a) Inspection of parts. (b) Reassembly of parts.

The purpose of the Rapid Solutions Learning Projects (RSLP) funded by the Office of Naval Research is to educate undergraduate engineering students using hands-on learning projects that solve problems recommended by the local ONR Science Advisors, the Naval Warfare Centers and Fleet Forces Commands. As part of the RSLP, this project will develop an augmented reality application for operation and maintenance of M16A4.

\section{Design and Implementation}

This project contains several major tasks: 1) 3D modeling of the weapon, including all internal and external parts, 2) 3D printing of the weapon mockup, and 3) AR software development in Unity. However, almost all universities ban weapons on campus and even if weapons are allowed for research purposes such as this project, safekeeping the weapon would be a logistical challenge. 3D printing is employed to create mockups of M16A4. One faculty advisor supervises a student on 3D modeling and 3D printing, while the other faculty advisor directs the second student on AR software development.

\subsection{D Modeling and Printing}

A digital 3D model of M16A4 is shown in Figure 2(a) and Forms 2 3D printer (Figure 2(b)) is utilized in this project to print the M16A4 mockup. 


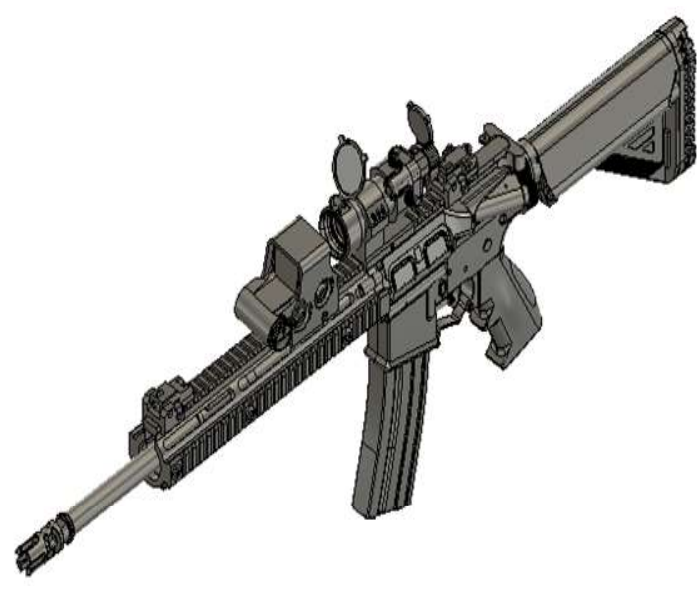

(a)

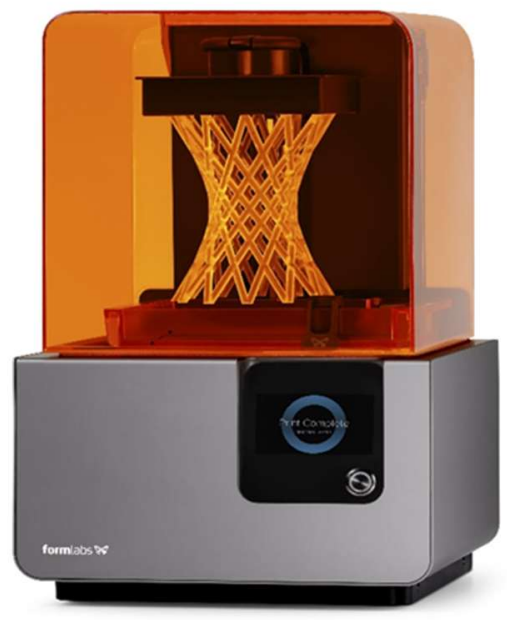

(b)

Figure 2. M16A4 Mockup 3D printing. (a) Digital model of M16A4. (b) Form 2 3D printer manufactured by Formlabs.

The M16A4's length exceeds Form 2's build volume. Therefore, the M16A4 must be dissembled into multiple components that can appropriately fit the Form 2 printer. Formlabs' software tool Preform allows the user to input a component, orient, size, and set the type of build supports to personal specifications. This procedure was repeated for every single piece of the gun, which was broken into 11 major components. The current assembled M16A4 is shown in Figure 3, which represents about $85 \%$ of the entire rifle.

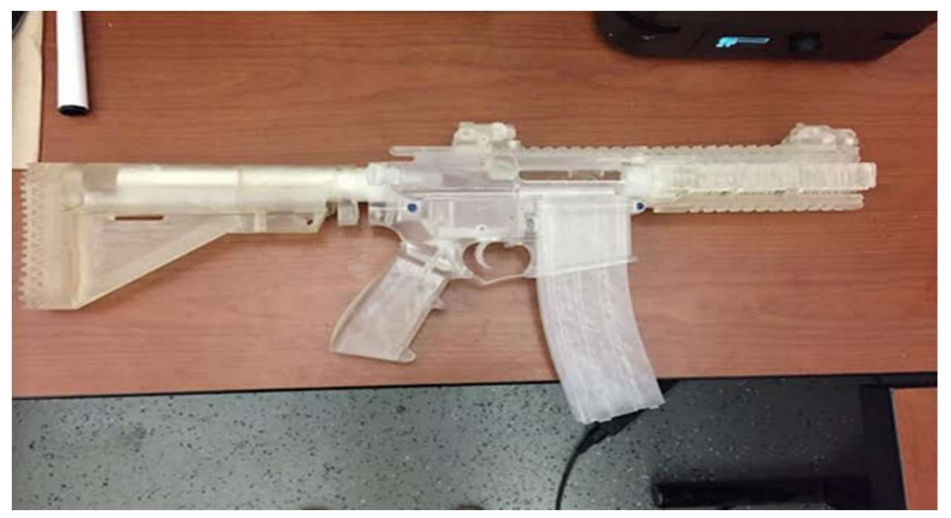

Figure 3. Current status of the M16A4 mockup

\subsection{AR Software Development}

Currently the state-of-the-art approach for developing AR applications is to utilize game engines, such as Unity [6, 7] and Unreal [7]. Game engines are integrated software development environments for building video games $[6,7,21,22]$. The core functionalities provided by a game engine usually include a rendering engine, a physics engine, scripting, animation, artificial intelligence, and cinematics, among other capabilities. These functionalities allow game developers to create quality games without the need of developing everything from scratch. In addition, most current generation game engines support cross-platform development, i.e., the same game code can be built and deployed to different hardware and software platforms with 
only minor modification of the code. Unity [6] is a leading game engine for developing games, simulations, virtual environments, virtual reality, and augmented reality applications, and is utilized in this project.

One critical aspect of AR application development is the recognition and tracking of real-world objects (M16A4 in this project) and then project information or virtual objects into the real world. Various techniques can be used to recognize and track objects, such as optical tracking, structured light, marker-based tracking, and model-based tracking [1,2]. Vuforia is a marketleading AR technology that utilizes advanced computer vision to recognize and track real-world objects using a variety of methods, such as images, markers (VuMarks), and multi-targets [8]. Vuforia is used in this project to develop the AR application for M16A4.

\section{Current Results}

A mobile version of the M16A4 application has been developed and deployed to Android and iOS platforms. Figure 4(a) shows the application user interface, which supports interactive visualizations (pan, orbit, and zoom) and various operation and maintenance procedures (part labelling, part highlighting, and maintenance). Figure 4(b) shows a zoomed, paned, and orbited view of the rifle. Figure 4(c) display part names of the rifle. Dynamic labelling of interactive $3 \mathrm{D}$ objects is a challenging and unresolved problem and substantial research is needed to produce effective and satisfactory results. Figure 4(d) shows a highlighted view of the scope of the rifle, while other parts are displayed in translucent mode.

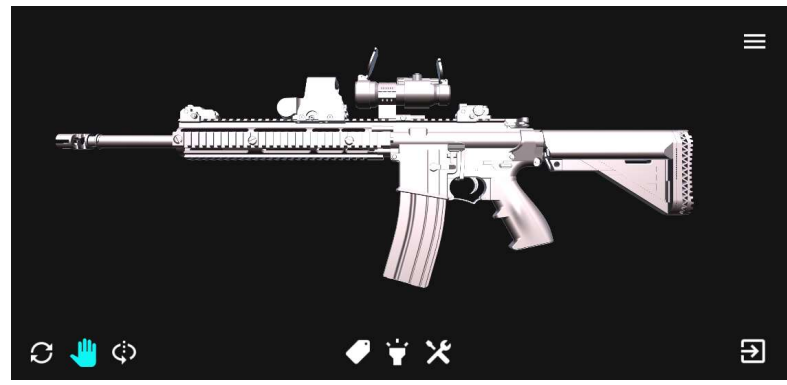

(a)

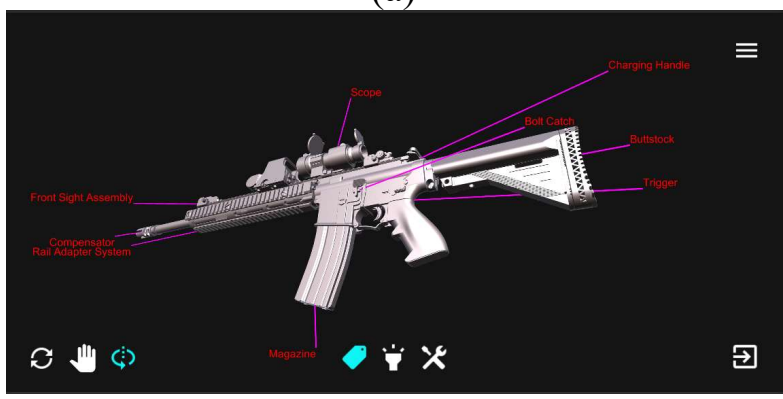

(c)

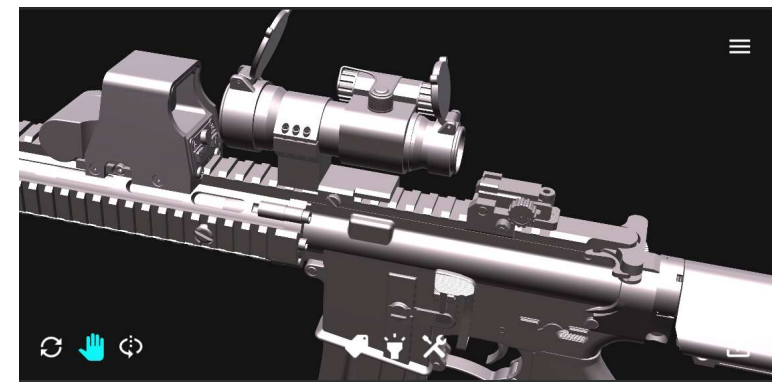

(b)

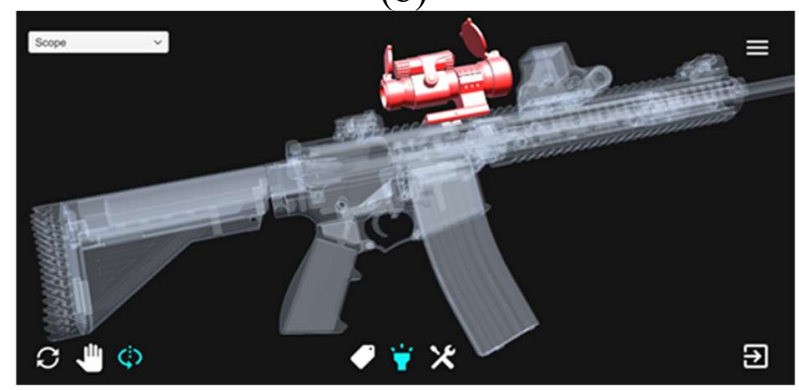

(d)

Figure 4. Screen captures of the current A16M4 AR application. (a) Software user interface. (b) Zoomed and panned view. (c) Label of the parts, and (d) Highlighted x-ray view.

Figure 5(a) shows the procedure for troubleshooting a malfunction (failure of magazine to lock in weapon) in the M16A4 manual [20]. The procedure consists of 4 steps that are displayed on a single page of the PDF manual. The M16A4 AR application, on the other hand, displays one 
step at a time, greatly reducing the cognitive load of the user. Figure 5(b) shows the $4^{\text {th }}$ step presented by the AR application. The suspected faulty parts are highlighted, and instructions are displayed closely to the parts in question.

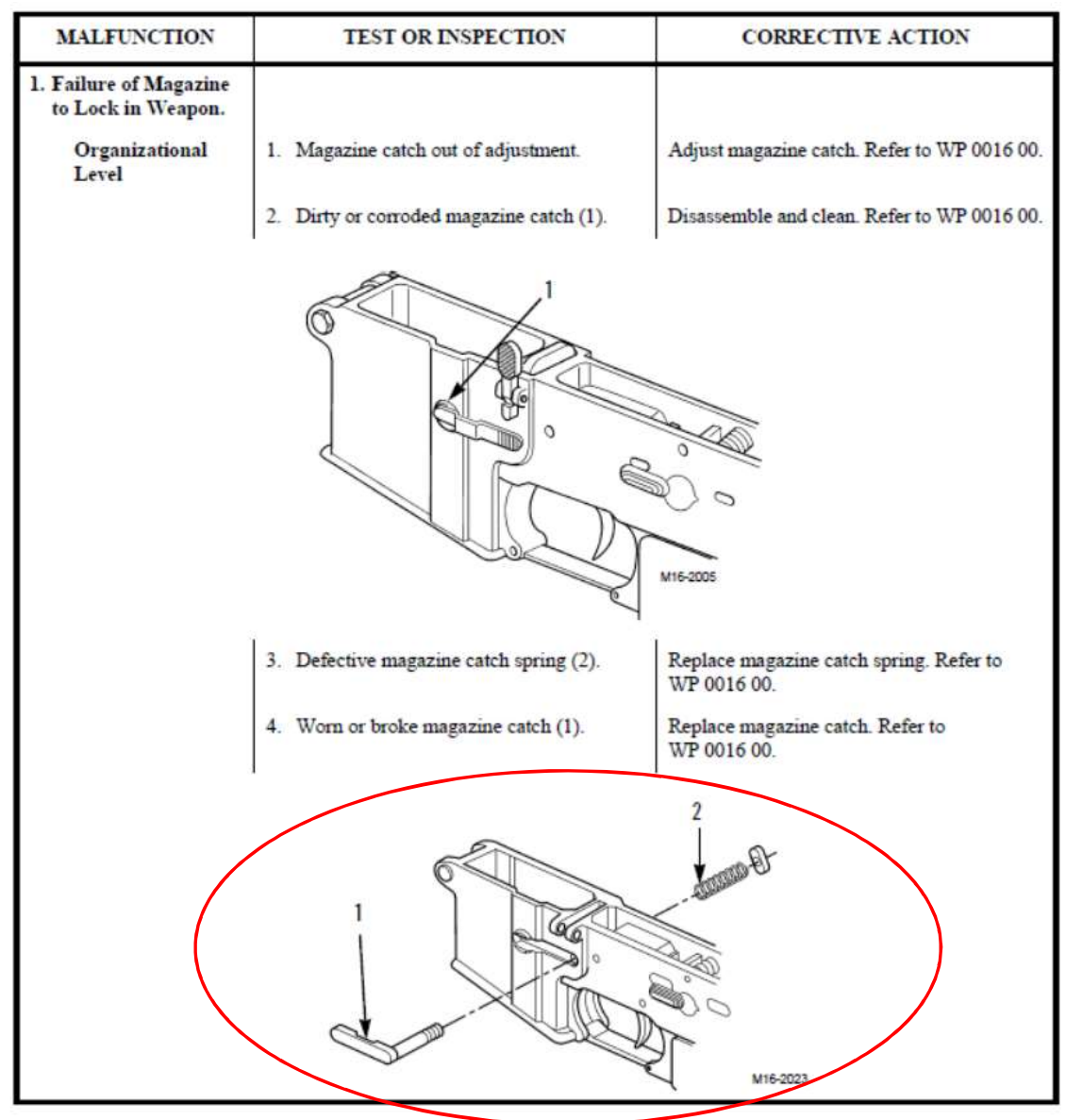

(a)

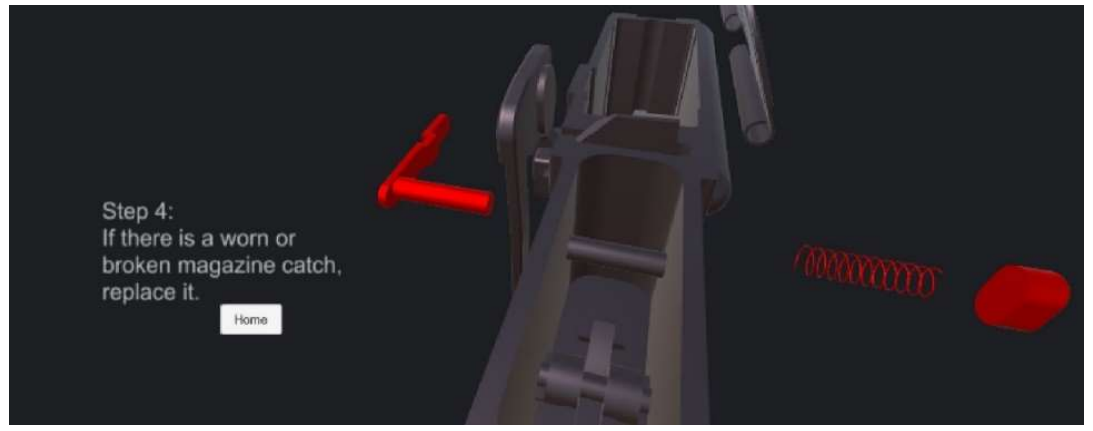

(b)

Figure 5. Troubleshooting for malfunction: failure of magazine to lock in weapon. (a) the instructions in the M16A4 manual [20]. (b) The $4^{\text {th }}$ step of the troubleshooting procedure presented by the AR application, which greatly reduces the cognitive load of the user.

The completed software will contain two modes: virtual reality mode (VR) and augmented reality mode (AR). Development of VR mode is complete with simple labelling. The 
development of AR mode with marker tracking is also complete with VuMarks. AR with multitarget (3D model) tracking will be integrated once the 3D rifle is fully printed. Initial experimentation with smaller $3 \mathrm{D}$ models demonstrates very promising results.

\section{Discussion and Conclusion}

Information representation and communication are two important aspects in this multidisciplinary project. For example, different file formats are required by different applications or purposes. The STL file format is commonly used for 3D printing, while FBX file format is the de facto standard for AR applications. In addition, these two file types contain different types of information. The STL files only contain geometry information of an object for 3D printing, while an FBX file contains additional attributes for graphical rendering, such as material colors and vertex normals. The initial conversion process from STL format to FBX format didn't produce correct vertex normals, resulting in incorrect display in the AR application that stalled the project progress for more than two weeks. The STL files were finally successfully converted to the FBX format after fixing the vertex normal. This demonstrates one issue in multidisciplinary projects, i.e., the same object can have different representations for different applications. While this caused delay, it does demonstrate one benefit of multidisciplinary projects once the problem is fixed. By solving this issue, students have better understanding of the inherent complex nature of real-world multidisciplinary projects and the importance of possessing relevant knowledge of other disciplines. Another related benefit is that students better understand the design and development process of other disciplines. One lesson learned from this project is that the importance of communication between different teams should be emphasized from the very beginning of the project and different teams should communicate more frequently (e.g., meeting).

The final version of the application will include the real (or Mockup of) M16A4, and the highlighted parts and instructions will be aligned with the real M16A4. Very promising results have been achieved, and continuing work includes object recognition and tracking using Vuforia and deployment to Microsoft HoloLens. Also, several difficulties and challenges remain. First, although 3D printing is utilized to create a mockup for the real weapon in this project, it is a very slow process and currently 3D printing technology is still not very reliable. Second, interactive 3D labeling is a difficult and unsolved problem; more substantial research beyond the scope of this undergraduate research project is needed. Third, although interactive visualization and presentations are very useful for operations and maintenance, translating the traditional manuals into interactive visualizations is a tedious process; more research is needed to expedite the translation process.

\section{References}

1. Schmalstieg, D. and T. Hollerer, Augmented Reality: Principles and Practice (Usability). 2016: Addison-Wesley Professional.

2. Aukstakalnis, S., Practical Augmented Reality: A Guide to the Technologies, Applications, and Human Factors for AR and VR (Usability). 2016: Addison-Wesley Professional.

3. Wikipedia. Pokemon Go. 2019; Available from: https://en.wikipedia.org/wiki/Pokémon_Go. 
4. Wang, W., Beginning ARKit for iPhone and iPad: Augmented Reality App Development for $i O S$. 2018: Apress.

5. Lanham, M., Learn ARCore - Fundamentals of Google ARCore: Learn to build augmented reality apps for Android, Unity, and the web with Google ARCore. 2018: Packt Publishing.

6. Unity Technologies. Unity. 2019; Available from: http://unity3d.com/unity/.

7. Shannon, T., Unreal Engine 4 for Design Visualization: Developing Stunning Interactive Visualizations, Animations, and Renderings (Game Design). 2017: Addison-Wesley Professional.

8. PTC Inc. Vuforia. 2019; Available from: https://www.vuforia.com/.

9. DAQRI. ARToolKit. 2019; Available from: https://github.com/artoolkit.

10. Zerhouni, E., et al., Training the Workforce for 21st Century Science. 2016, National Academy of Medicine: National Academy of Medicine.

11. Committee on Improving Higher Education's Responsiveness to STEM Workforce Needs: Identifying Analytical Tools and Regional Best Practices, et al., Promising Practices for Strengthening the Regional STEM Workforce Development Ecosystem. 2016: National Academy of Sciences.

12. Committee on Revitalizing Graduate STEM Education for the 21st Century, et al., Graduate STEM Education for the 21st Century, ed. A. Leshner and L. Scherer. 2018: The National Academies Press.

13. Committee on Integrating Higher Education in the Arts, Humanities, Sciences, Engineering, and Medicine, Board on Higher Education and Workforce, Policy and Global Affairs, and National Academies of Sciences, Engineering, and Medicine, The Integration of the Humanities and Arts with Sciences, Engineering, and Medicine in Higher Education: Branches from the Same Tree, ed. D. Skorton and A. Bear. 2018: The National Academies Press.

14. Alper, J., et al., Developing a National STEM Workforce Strategy: A Workshop Summary. 2016: The National Academies Press.

15. National Science Board, Science \& Engineering Indicators 2018. 2018, National Science Board.

16. Committee on Science, Technology, Engineering, and Mathematics Workforce Needs for the U.S. Department of Defense and the U.S. Defense Industrial Base, N.A.o. Engineering, and N.R. Council, Report of a Workshop on Science, Technology, Engineering, and Mathematics (STEM) Workforce Needs for the U.S. Department of Defense and the U.S. Defense Industrial Base. 2012: The National Academies Press.

17. National Science Foundation. Dear Colleague Letter: STEM Workforce Development Utilizing Flexible Personal Learning Environments. 2019; Available from: https://www.nsf.gov/pubs/2019/nsf19025/nsf19025.jsp.

18. ABET. Criteria for Accrediting Engineering Programs, 2018 - 2019. 2019; Available from: https://www.abet.org/accreditation/accreditation-criteria/criteria-for-accreditingengineering-programs-2018-2019/\#GC1.

19. US Marine Corps, Maintenance Operations, Department of Defense, Editor. 2016.

20. U.S. Marine Corps, Organizational And Intermediate Maintenance Manual With Repair Parts List (RPL) For M16A2, M16A4, M4, And M4A1. 2008.

21. Gregory, J., J. Lander, and M. Whitting, Game Engine Architecture. 2018: A. K. Peters.

22. Lengyel, E., Game Engine Gems 3. 2016: A K Peters/CRC Press. 